\title{
A Comprehensive Analysis of 5-Year Outcomes in Patients with Cancer Admitted to Intensive Care Units
}

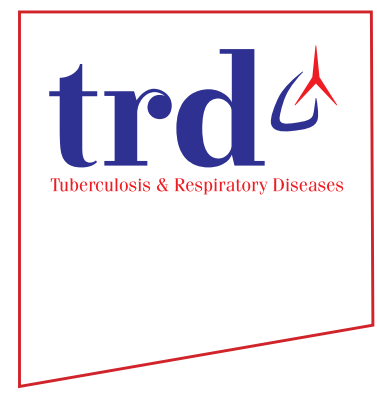

\author{
Yoonki Hong, M.D., Ph.D. ${ }^{1}{ }^{\mathbb{D}}$, Woo Jin Kim, M.D., Ph.D. ${ }^{1}$, Ji Young Hong, M.D., Ph.D. ${ }^{2}$, Yun-jeong \\ Jeong, M.D., M.S. ${ }^{3}$ and Jinkyeong Park, M.D., Ph.D. ${ }^{3, \dagger}$ \\ ${ }^{1}$ Department of Internal Medicine, Kangwon National University Hospital, Kangwon National University School of Medicine, \\ Chuncheon, ${ }^{2}$ Division of Pulmonary and Critical Care Medicine, Department of Medicine, Chuncheon Sacred Heart Hospital, \\ Hallym University Medical Center, Chuncheon, ${ }^{3}$ Department of Internal Medicine, Dongguk University Ilsan Hospital, Goyang, \\ Republic of Korea
}

Background: The aim of this study was to evaluate the long-term (5-year) clinical outcomes of patients who received intensive care unit (ICU) treatment using Korean nationwide data.

Methods: All patients aged $>18$ years with ICU admission according to Korean claims data from January 2008 to December 2010 were enrolled. These enrolled patients were followed up until December 2015. The primary outcome was ICU mortality.

Results: Among all critically ill patients admitted to the ICU $(n=323,765)$, patients with cancer showed higher ICU mortality $(18.6 \%)$ than those without cancer $(13.2 \%$, $\mathrm{p}<0.001)$. However, there was no significant difference in ICU mortality at day 28 among patients without cancer (14.5\%) and those with cancer (lung cancer or hematologic malignancies) (14.3\%). Compared to patients without cancer, hazard ratios of those with cancer for ICU mortality at 5 years were: 1.90 (1.87-1.94) for lung cancer; $1.44(1.43-1.46)$ for other solid cancers; and 3.05 (2.95-3.16) for hematologic malignancies.

Conclusion: This study showed that the long-term survival rate of patients with cancer was significantly worse than that of general critically ill patients. However, short term outcomes of critically ill patients with cancer were not significantly different from those of general patients, except for those with lung cancer or hematologic malignancies.

Keywords: Neoplasms; Intensive Care Unit; Mortality; Hematologic Malignancies; Lung Neoplasms; Solid Cancer

Address for correspondence: Jinkyeong Park, M.D., Ph.D.

Department of Internal Medicine, Dongguk University Ilsan Hospital, 27 Dongguk-ro, Ilsandong-gu, Goyang 10326, Republic of Korea

Phone: 82-31-961-7969

E-mail: driinnie@me.com

'Present address: Department of Pulmonary, Allergy and Critical Care Medicine, Kyung Hee University Hospital at Gangdong, Kyung Hee

University School of Medicine, Seoul, Republic of Korea

Received: Jul. 7, 2021

Revised: Nov. 3, 2021

Accepted: Jan. 16, 2022

Published online: Jan. 20, 2022

(c) It is identical to the Creative Commons Attribution Non-Commercial License (http://creativecommons.org/licenses/by-nc/4.0/).

The Korean Academy of Tuberculosis and Respiratory Diseases.

\section{Introduction}

The incidence rate of cancer is increasing as the elderly population increases. The National Institutes of Health (Bethesda, MD, USA $)^{1}$ has estimated that approximately $15,760,939$ individuals have cancer in the United States of America. The Korea Central Cancer Registry ${ }^{2}$ has reported that one in then elderly individuals aged $>65$ years have cancer. Early detection of cancer and advances in treatment options are increasing the overall cancer survival rate ${ }^{3}$. Nevertheless, as the number of patients with cancer increases, so does the number of patients requiring admission to the intensive care unit (ICU) ${ }^{4}$. Taccone et al. ${ }^{5}$ have reported that one in seven critically ill patients admitted to the general ICU in Europe is a patient with cancer. 
Numerous recent studies ${ }^{6-8}$ have reported a decrease in mortality despite an increase in the number of patients admitted to the ICU as well as an increase in severity or comorbidities. Many studies have shown that ICU admission can help patients after receiving urgent chemotherapy or undergoing hematopoietic stem cell transplantation, in whom many adverse outcomes have been previously recorded ${ }^{9-11}$. This is because considerable effort has been focused on providing early critical care to select patients with cancer ${ }^{12,13}$. However, most of these studies assessed relatively short-term outcomes. Numerous limitations characterize the short-term outcome assessment in clarifying the criteria for treatment continuation, appropriate ICU utilization, and precise indications for ICU admission ${ }^{14}$. Further investigation of long-term outcomes is warranted to reduce the uncertainty in critically ill patients.

Thus, the aim of this study was to investigate long-term outcomes depending on the type of cancer. This populationbased cohort study compared 5-year mortality rates of critically ill patients with and without cancer to investigate differences in the long-term outcome of each type of cancer.

\section{Materials and Methods}

\section{Data source}

This was a retrospective observational cohort study using claims data from the Health Insurance Review and Assessment Service (HIRA) between January 1, 2007 and December 31,2015 . In Korea, all individuals are covered by the National Health Insurance or Medical Aid Program. The claims data contain information regarding patients' diagnoses, treatments, procedures, surgical history, and use of prescription drugs.

\section{Patients}

The study population included all patients aged $>18$ years who were examined by chest computed tomography for any reasons from January 2007 to December 2012. Enrolled patients were followed up until March 31, 2016. We identified the first ICU admissions with codes of ICU services (modified version of the International Classification of Diseases 10th revision [ICD-10] codes: AJ001-AJ590900). Patients with ICU admission were divided into two groups based on the presence or absence of cancer since January 2008. Exclusion

Table 1. Characteristics and outcomes of critically ill patients

\begin{tabular}{|c|c|c|c|c|}
\hline Characteristic & $\begin{array}{c}\text { Total } \\
(n=323,765)\end{array}$ & $\begin{array}{l}\text { With cancer } \\
(n=87,936)\end{array}$ & $\begin{array}{l}\text { Without cancer } \\
(n=235,829)\end{array}$ & p-value \\
\hline Female sex & $128,471(39.7)$ & $28,611(32.5)$ & $99,860(42.3)$ & $<0.001$ \\
\hline Age, yr & $66.2 \pm 14.4$ & $66.0 \pm 12.3$ & $66.3 \pm 15.1$ & $<0.001$ \\
\hline Charlson Comorbidity Index & $3.1 \pm 2.8$ & $5.5 \pm 3.1$ & $2.2 \pm 2.0$ & $<0.001$ \\
\hline Overall follow-up, day & $1,074.7 \pm 988.6$ & $843.6 \pm 908.9$ & $1,160.9 \pm 1,003.2$ & $<0.001$ \\
\hline LOS in ICU, day & $16.2 \pm 14.3$ & $18.2 \pm 14.5$ & $15.4 \pm 14.2$ & $<0.001$ \\
\hline Use of mechanical ventilator & $104,444(32.3)$ & $26,222(29.8)$ & $78,222(33.2)$ & $<0.001$ \\
\hline Renal replacement therapy & $21,261(6.0)$ & $4,903(5.6)$ & $16,358(6.9)$ & $<0.001$ \\
\hline CRRT & $11,974(3.7)$ & $3,305(3.8)$ & $8,669(3.7)$ & 0.273 \\
\hline Vasopressor & $100,836(31.1)$ & $26,996(30.7)$ & $73,840(31.3)$ & 0.001 \\
\hline Overall mortality & $176,202(54.4)$ & $55,388(63.0)$ & $120,814(51.2)$ & $<0.001$ \\
\hline ICU mortality & $47,459(14.7)$ & $16,388(18.6)$ & $341,071(13.2)$ & $<0.001$ \\
\hline ICU mortality at day 28 & $50,069(15.5)$ & $15,959(18.1)$ & $34,110(14.5)$ & $<0.001$ \\
\hline ICU mortality at day 60 & $68,747(21.2)$ & $23,008(26.2)$ & $45,739(19.4)$ & $<0.001$ \\
\hline ICU mortality at day 90 & $77,585(24.0)$ & $26,164(29.8)$ & $51,421(21.8)$ & $<0.001$ \\
\hline ICU mortality at 6 months & $92,314(28.5)$ & $31,349(35.6)$ & $60,965(25.9)$ & $<0.001$ \\
\hline ICU mortality at 1 year & $109,618(33.9)$ & $37,526(42.7)$ & $72,093(30.6)$ & $<0.001$ \\
\hline ICU mortality at 2 years & $130,656(40.4)$ & $44,654(50.8)$ & $86,002(36.5)$ & $<0.001$ \\
\hline ICU mortality at 3 years & $145,106(44.8)$ & $48,662(55.3)$ & $96,444(40.9)$ & $<0.001$ \\
\hline ICU mortality at 5 years & $166,595(51.5)$ & $53,585(60.9)$ & $113,010(47.9)$ & $<0.001$ \\
\hline
\end{tabular}

Values are presented as number $(\%)$ or mean \pm SD.

LOS: length of stay; ICU: intensive care unit; CRRT: continuous renal replacement therapy; SD: standard deviation. 
criteria were as follows: (1) patients aged >100 years; (2) ICU admission prior to the diagnosis of cancer; and (3) any claims related to cancer prior to January 2008. Cancer was categorized into lung cancer (code C34 of the ICD-10), hematologic malignancy (codes C81-C86, C88, and C90-C95 of the ICD10), and other solid cancers.

\section{Comorbidities and concomitant medical therapy}

Comorbidities were recognized if claims data existed 6 months before the index of admission to the ICU. Comorbidity diagnoses were reached using ICD-10 codes. Concomitant medical therapy was defined by the procedure code of the Korean National Health Insurance or Korean drug and anatomical therapeutic chemical codes.

\section{Ethics}

This study was approved by the HIRA. Ethical approval for this study was exempted by the Kangwon National University Hospital Institutional Review Board (B-2018-02-002) because the authors only accessed de-identified, previously collected data.

A

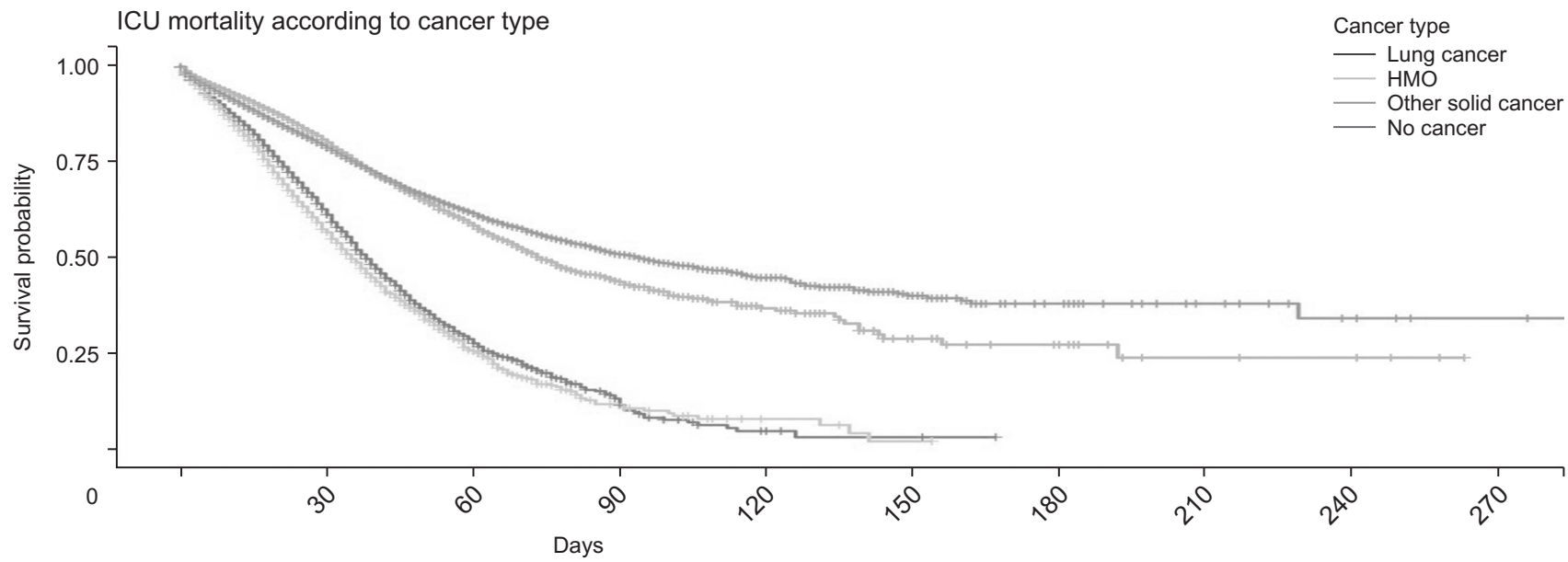

$\begin{array}{rcc}\text { No. at risk } & & \\ \text { Lung cancer } & 20,902 & 2,709 \\ \text { HMO } & 4,552 & 1,348 \\ \text { Other solid cancer } & 53,282 & 9,362 \\ \text { No cancer } & 235,829 & 28,346\end{array}$

B

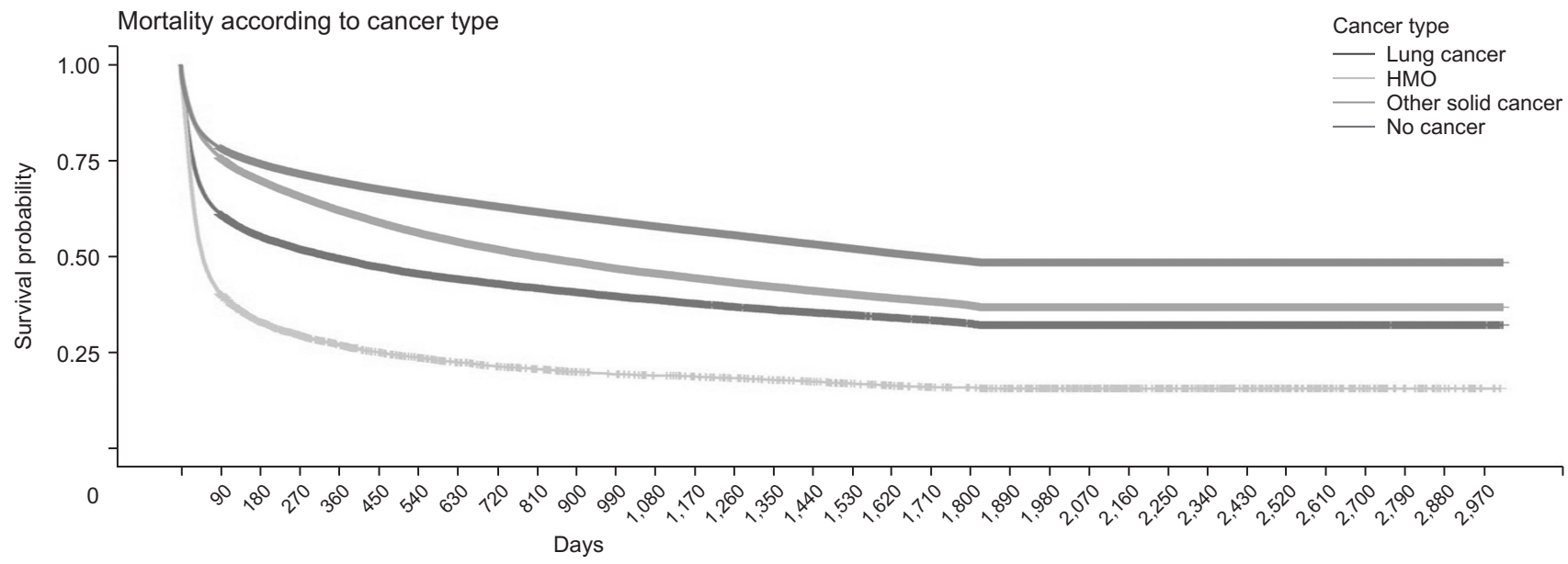

No. at risk

Lung cancer

$\mathrm{HMO}$

Other solid cancer

No cancer

$\begin{array}{lllllllllllllllllllllllllllllllllll}20,902 & 12,781 & 11,328 & 10,423 & 9,666 & 9,061 & 8,578 & 8,196 & 7,840 & 7,507 & 7,226 & 6,938 & 6,658 & 6,377 & 6,122 & 5,883 & 5,669 & 5,427 & 5,208 & 5,008 & 4,764 & 4,444 & 3,955 & 3,488 & 2,995 & 2,561 & 2,201 & 1,335 & 1,469 & 1,154 & 843 & 573 & 312 & 64\end{array}$ $\begin{array}{llllllllllllllllllllllllllllllllllll}4,552 & 1,838 & 1,453 & 1,243 & 1,081 & 970 & 889 & 816 & 761 & 713 & 668 & 640 & 614 & 594 & 566 & 534 & 505 & 469 & 438 & 410 & 397 & 372 & 338 & 290 & 242 & 211 & 175 & 138 & 109 & 81 & 52 & 35 & 16 & 3\end{array}$ $\begin{array}{llllllllllllllllllllllllllllllllllll}53,282 & 40,395 & 36,323 & 33,288 & 30,573 & 28,272 & 26,351 & 24,628 & 23,183 & 21,799 & 20,627 & 19,488 & 18,456 & 17,462 & 16,538 & 15,669 & 14,776 & 13,977 & 13,214 & 12,541 & 11,793 & 10,991 & 9,870 & 8,605 & 7,403 & 6,276 & 5,258 & 4,292 & 3,393 & 2,581 & 1,359 & 1,243 & 661 & 155\end{array}$

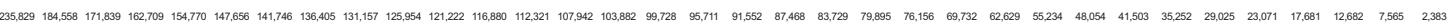

Figure 1. Kaplan-Meier curves of risk for all-cause death in intensive care unit (ICU) (A) and at 5 years of each group (B) compared with that of critically ill patients without cancer. HMO: hematology and medical oncology. 


\section{Statistical analysis}

The study endpoint was all-cause mortality in the ICU. Variables are presented as numbers (percentages) or means (standard deviations). Between-group comparisons were performed using $\chi^{2}$ tests for categorical data and Student's t-tests for continuous data. Cox proportional hazards regression was used to calculate the hazard ratios (HRs) and 95\% confidence intervals (CIs) for in-hospital mortality associated with patient characteristics. The value obtained by subtracting the cancerrelated score from the Charlson Comorbidity Index (CCI) system was used to avoid overcorrection of cancer disease. Probabilities $<0.05$ were considered statistically significant. All analyses were performed using R v3.4.4 with packages of survival and ggplot2 function in R to obtain an appropriate updated citation.

\section{Results}

During the study period, 323,765 patients were admitted to the ICU at least once. Of all critically ill patients admitted to the ICU, the proportion of patients with cancer continued to increase from $21.5 \%$ in 2008 to $27.8 \%$ in 2010 . Critically ill patients with cancer showed significant male predominance $(67.5 \%, \mathrm{p}<0.001)$ and higher CCI (5.5 \pm 3.1 vs. $2.2 \pm 2.0, \mathrm{p}<0.001)$ than patients without cancer (Table 1). The length of stay in the ICU was significantly longer for patients with cancer than for those without cancer $(18.2 \pm 14.5$ days vs. $15.4 \pm 14.2$ days, $\mathrm{p}<0.001)$. Patients with cancer in the ICU received less support by mechanical ventilator than those without cancer $(29.8 \%$ vs. $33.2 \%, \mathrm{p}<0.001)$. Critically ill patients with cancer showed higher ICU mortality than those without cancer (18.6\% vs. $13.2 \%, \mathrm{p}<0.001)$. Following admission to the ICU, mortality rates of patients with cancer were $18.1 \%, 26.2 \%, 42.7 \%$, and $55.3 \%$ at 28 days, 60 days, 1 year, and 3 years, respectively (Figure 1). There was no significant difference in ICU mortality at day 28 between patients without cancer and those with cancer (not lung cancer or hematologic malignancies) (14.5\% vs. $14.3 \%, \mathrm{p}=0.28$ ). However, the difference was statistically significant thereafter. After adjusting for age, sex, year of admission to the ICU, and use of life support system (such as ventilator, continuous renal replacement therapy, and vasopressor), the HR of ICU mortality in all patients with cancer at 5 years was 1.56 (95\% CI, 1.53-1.59) versus those without cancer. However, there was no significant difference in the HR of ICU mortality for patients with cancer which was not lung cancer or hematologic malignancy versus those without cancer (HR, 1.00; 95\% CI, 0.998-1.016) (Figure 2).

\section{Comparison between patients with solid cancer and hematologic malignancy}

Of 78,736 patients with cancer, except for 9,200 patients (10.5\%) with multiple primaries, $5.8 \%$ had hematologic malignancies. The proportion of patients with a hematologic malig-

Mortality of each group compared with critically ill patients without cancer

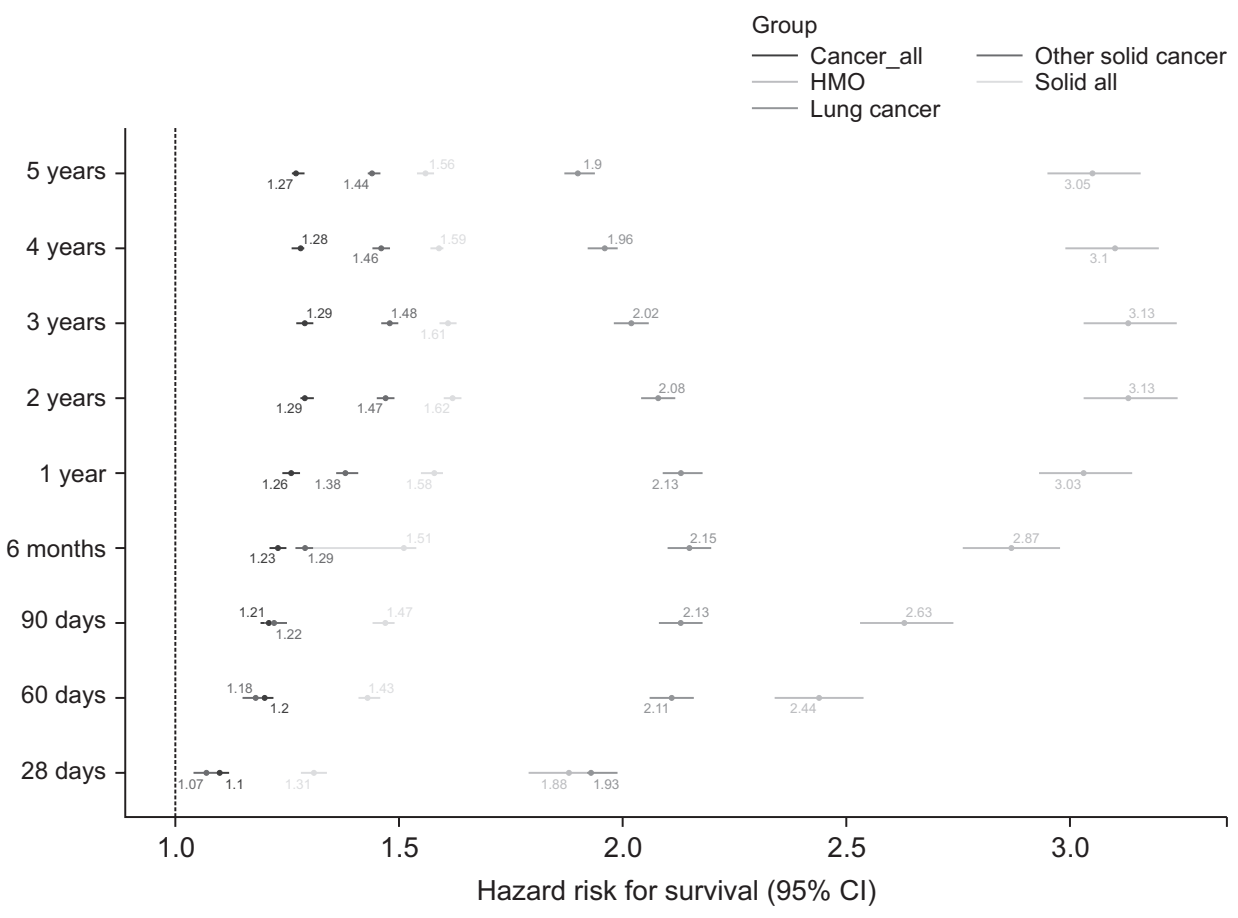

Figure 2. Cox regression proportional hazard model analysis of all-cause mortality in comparison with critically ill patients without cancer. Adjusted hazard ratios (solid circles) and $95 \%$ confidence intervals (horizontal lines) for death are shown. Hazard ratios were adjusted for age, sex, year of admission to the intensive care unit, and use of life support system (such as ventilator, continuous renal replacement therapy, and vasopressor). CI: confidence interval; HMO: hematology and medical oncology. 
nancy among critically ill patients with cancer increased from $5.4 \%$ in 2008 to $6.6 \%$ in 2010 . These patients were predominantly females, younger, and had lower CCI versus those with solid cancer (Table 2). Patients with hematologic malignancy stayed longer in the ICU ( $22.7 \pm 17.3$ days) than those with solid cancer (18.1 \pm 4.3 days). The use of medical support and life support procedures was more frequent in critically ill patients with a hematologic malignancy than in those with solid cancer (use of mechanical ventilators: $28.6 \%$ vs. $54.5 \%$, $\mathrm{p}<0.01$; use of renal replacement therapy: $4.8 \%$ vs. $22.0 \%$, $p<0.01$ ). The ICU mortality was higher in patients with hematologic malignancy than in those with solid cancer (43.9\% vs. $17.5 \%$, p $<0.01$ ) (Figure 1). The hazard risk of hematologic malignancies and lung cancer on day 28 was almost the same as that of general patients in the ICU (Figure 2). Compared with patients without cancer, HRs of ICU mortality were 1.49 (95\% CI, 1.46-1.52) and 2.37 (95\% CI, 2.15-2.28) in patients with solid cancer and hematologic malignancies, respectively.

\section{Comparison within solid cancer: lung cancer versus other solid cancers}

Lung cancer accounted for $28.2 \%$ of critically ill patients with solid cancer. Patients with lung cancer had a higher percentage of males ( $72.4 \%$ vs. $65.8 \%$, $\mathrm{p}<0.001)$ than those with other solid cancers. Patients with lung cancer were admitted sooner to the ICU following diagnosis than patients with other solid cancers $(205.1 \pm 409.5$ days vs. $357.1 \pm 571.0$ days, $\mathrm{p}<0.001)$. Patients with lung cancer had a shorter length of stay in the ICU than those with other solid cancers $(16.1 \pm 12.9$ days vs. $18.8 \pm 14.6$ days, $\mathrm{p}<0.001)$. In addition, they required more ventilator support than others (31.0\% vs. $27.6 \%, \mathrm{p}<0.01)$. ICU mortality was higher in patients with lung cancer than in those with other solid cancer $(25.5 \%$ vs. $14.4 \%$, p $<0.01)$. Compared with patients without cancer, the HRs of 28-day and 5-year survival were 1.93 (95\% CI, 1.87-1.99) and 1.90 (95\% CI, 1.87-1.94) in patients with lung cancer and 1.07 (95\% CI, 1.04-1.10) and 1.44 (95\% CI, 1.43-1.46) in patients with other solid cancers, respectively (Figure 2).

Table 2. Characteristics and outcomes of critically ill patients according to cancer type except for multiple primaries

\begin{tabular}{|c|c|c|c|c|}
\hline \multirow[b]{2}{*}{ Characteristic } & \multicolumn{2}{|c|}{ Solid cancer } & \multirow{2}{*}{$\begin{array}{c}\text { Hematologic } \\
\text { malignancies } \\
(n=4,552)\end{array}$} & \multirow[b]{2}{*}{ p-value } \\
\hline & $\begin{array}{l}\text { Lung cancer } \\
(n=20,902)\end{array}$ & $\begin{array}{l}\text { Other solid cancers } \\
\qquad(n=53,282)\end{array}$ & & \\
\hline Female sex & $5,770(27.6)$ & $18,247(34.2)$ & $1,879(41.3)$ & $<0.001$ \\
\hline Age, yr & $67.3 \pm 10.8$ & $66.0 \pm 12.5$ & $60.4 \pm 15.3$ & $<0.001$ \\
\hline Charlson Comorbidity Index & $5.5 \pm 3.1$ & $5.5 \pm 3.1$ & $4.3 \pm 2.6$ & $<0.001$ \\
\hline Interval from diagnosis to ICU & $205.1 \pm 409.5$ & $357.1 \pm 571.0$ & $320.7 \pm 514.4$ & $<0.001$ \\
\hline Overall follow-up & $796.2 \pm 946.9$ & $887 \pm 896.4$ & $383.1 \pm 693.2$ & $<0.001$ \\
\hline LOS in ICU & $16.1 \pm 12.9$ & $18.8 \pm 14.7$ & $22.7 \pm 17.3$ & $<0.001$ \\
\hline Use of mechanical ventilator & $6,490(31.0)$ & $14,715(27.6)$ & $2,483(54.5)$ & $<0.001$ \\
\hline Renal replacement therapy & $660(3.2)$ & $2,878(5.4)$ & $1,002(22.0)$ & $<0.001$ \\
\hline CRRT & $450(2.2)$ & $1,793(3.4)$ & $792(17.4)$ & 0.273 \\
\hline Vasopressor & $6,017(28.8)$ & $15,799(29.7)$ & $2,693(59.2)$ & 0.001 \\
\hline Overall mortality & $13,976(66.9)$ & $31,831(59.7)$ & $3,736(82.1)$ & $<0.001$ \\
\hline ICU mortality & $5,322(25.5)$ & $7,676(14.4)$ & $1,997(43.9)$ & $<0.001$ \\
\hline ICU mortality at day 28 & $5,291(25.3)$ & $7,609(14.3)$ & $1,638(36.0)$ & $<0.001$ \\
\hline ICU mortality at day 60 & $7,363(35.2)$ & $11,136(20.9)$ & $2,454(53.9)$ & $<0.001$ \\
\hline ICU mortality at day 90 & $8,145(39.0)$ & $12,928(24.3)$ & $2,722(59.8)$ & $<0.001$ \\
\hline ICU mortality at 6 months & $9,347(44.7)$ & $16,053(30.3)$ & $3,051(67.0)$ & $<0.001$ \\
\hline ICU mortality at 1 year & $10,551(50.5)$ & $20,125(37.8)$ & $3,311(72.7)$ & $<0.001$ \\
\hline ICU mortality at 2 years & $11,796(56.4)$ & $24,951(46.8)$ & $3,522(77.4)$ & $<0.001$ \\
\hline ICU mortality at 3 years & $12,548(60.0)$ & $27,599(51.8)$ & $3,602(79.1)$ & $<0.001$ \\
\hline ICU mortality at 5 years & $13,568(64.9)$ & $30,710(57.6)$ & $3,699(81.3)$ & $<0.001$ \\
\hline
\end{tabular}

Values are presented as number $(\%)$ or mean \pm SD.

ICU: intensive care unit; LOS: length of stay; CRRT: continuous renal replacement therapy; SD: standard deviation. 


\section{Discussion}

This study comprehensively presented short-term and long-term outcomes of critically ill patients over 5 years with relatively representative data. In the short term, outcomes of critically ill patients with cancer were not significantly different from those of general patients except for those with lung cancer or hematologic malignancies. After 60 days, the survival rate of patients with cancer was significantly worse than that of general critically ill patients. Although critical care had a limited effect on the overall course of cancer, it could be beneficial to patients with cancer in terms of overcoming the acute crisis in the ICU. However, patient selection for ICU admission is necessary for those with lung or hematologic malignancy who require intensive care to overcome an acute crisis.

Between 1987 and 1992, the 5-year survival rate of the Finnish mixed ICU was $59.9 \%{ }^{15}$. This finding revealed that cancer was a powerful factor in determining outcomes as bad as 3.17 of relative risk for death. Our data showed that, although the present study was conducted 10 years later, the 5 -year survival rate was lower than that of the Finnish ICU because the proportion of cancer patients was more than two-fold and the proportion of people with respiratory failure was more than four-fold. Moreover, our patients stayed 16.2 \pm 14.3 days in the ICU. According to Hermans et al. ${ }^{16}$, these patients can be termed "prolonged stayers." In this group of patients, the 5 -year survival rate was $51.8 \%$. Moreover, there was a difference of 5 years between studies. The previous research was conducted for 5 years since 2012, while ours was conducted 5 years earlier. In fact, the heterogeneity of patients observed between these studies complicated data comparisons.

Similar to the Finnish data ${ }^{15}$, our results demonstrated that critically ill patients with cancer had worse long-term outcomes than those without cancer. However, in our study, the short-term outcomes in the ICU (28 days) for patients with solid cancer (excluding lung cancer) were similar to those of general patients. The hazard risks for mortality within 28 days in patients with lung cancer and hematologic malignancies were similar. After 60 days, a difference in the survival rate and hazard risk for mortality became noticeable between these groups in the ICU. Survival rates of patients with other solid cancer (excluding lung cancer) showed a pattern closer to that of patients with lung cancer. Between 28 days and 180 days, the survival rate declined rapidly. Three years after admission to the ICU, patients with all types of cancer reached the life expectancy of critically ill patients without cancer. We found that, due to advancement of early cancer diagnosis technology enabling accurate cancer treatment and the development of the ICU, short-term outcomes of patients with cancer were markedly improved. Nevertheless, long-term outcomes for 5 years were 1.56 times higher in critically ill patients with cancer than in those without cancer.
Our study followed up critically ill patients for a long period of 5 years. However, a few limitations should be considered when interpreting our data. Firstly, this study used claims data designed for reimbursement purposes. It was not possible to present these data with standard scoring such as the Acute Physiology and Chronic Health Evaluation II or Simplified Acute Physiology Score. Alternatively, the condition of organ failure was assessed based on medical resources used for organ failure. There was no information on the performance status before admission to the ICU. This study might have included both low-severity patients and futile cases. Although it is difficult to identify accurate predictors for survival in this study, it is important to realistically assess the demand for ICU in a society where the number of patients with cancer increases. This is because it is difficult for physicians to actually deny admission to the ICU for patients with acute problems ${ }^{17}$. Secondly, we enrolled patients admitted to the ICU based on computed tomography examination. Hence, the present study might have included relatively limited data on patients with gastrointestinal alone or genitourinary alone problems. However, this might have little impact on overall outcomes of patients with solid cancer considering that outcomes of ICU care for most solid cancers except lung cancer were similar.

The policy of admission to the ICU for patients with cancer remains unclear. This is because most previous studies have reported predictors for survival based on short-term outcomes of less-representative populations rather than all patients with cancer. Our study of a relatively representative population can assist physicians in developing a comprehensive understanding of the relatively long-term prognosis of 5 years. In the future, more comprehensive research and interdisciplinary discussions are warranted. Communication and collaboration between the ICU team and oncologists and palliative care specialists should pre-describe clearer ICU admission criteria for patients with cancer and assist in making informed decisions.

\section{Author's Contributions}

Conceptualization: Hong Y, Hong JY, Park J. Methodology: Hong Y, Hong JY, Park J. Formal analysis: Hong Y, Hong JY, Park J. Data curation: Hong Y, Hong JY, Park J. Software: Park J. Investigation: Hong Y, Hong JY, Park J. Writing - original draft preparation: Hong Y, Park J. Writing - review and editing: Hong Y, Kim WJ, Jeong Y, Hong JY, Park J. Approval of final manuscript: all authors.

\section{Conflicts of Interest}

No potential conflict of interest relevant to this article was reported. 


\section{Funding}

This study was supported by a grant (NRF 2020R1A2C 1011455) of the National Research Foundation (NRF) funded by the Korean Government and a grant of the Korea Health Technology R\&D Project through the Korea Health Industry Development Institute (KHIDI), funded by the Ministry of Health \& Welfare, Republic of Korea (grant number: HI21C1074).

\section{References}

1. Surveillance Epidemiology and End Results Program, National Cancer Institute. Cancer stat facts: vancer of any site [Internet]. Bethesda: National Cancer Institute; 2020 [cited 2020 Sep 13]. Available from: https://seer.cancer.gov/statfacts/html/all.html.

2. Ministry of Health and Welfare. Main causes of mortality and incidence of major cancer types [Internet]. Sejong: Ministry of Health and Welfare; 2020 [cited 2020 Sep 13]. Available from: http://www.mohw.go.kr/react/gm/sgm0701ls.jsp?PAR_ MENU_ID=13\&MENU_ID=1304080402.

3. Brenner H. Long-term survival rates of cancer patients achieved by the end of the 20th century: a period analysis. Lancet 2002;360:1131-5.

4. van Vliet M, Verburg IW, van den Boogaard M, de Keizer NF, Peek N, Blijlevens NM, et al. Trends in admission prevalence, illness severity and survival of haematological patients treated in Dutch intensive care units. Intensive Care Med 2014;40:1275-84.

5. Taccone FS, Artigas AA, Sprung CL, Moreno R, Sakr Y, Vincent JL. Characteristics and outcomes of cancer patients in European ICUs. Crit Care 2009;13:R15.

6. Sauer CM, Dong J, Celi LA, Ramazzotti D. Improved survival of cancer patients admitted to the intensive care unit between 2002 and 2011 at a U.S. teaching hospital. Cancer Res Treat 2019;51:973-81.

7. Ostermann M, Ferrando-Vivas P, Gore C, Power S, Harrison D.
Characteristics and outcome of cancer patients admitted to the ICU in England, Wales, and Northern Ireland and national trends between 1997 and 2013. Crit Care Med 2017;45:166876.

8. Azoulay E, Soares M, Darmon M, Benoit D, Pastores S, Afessa B. Intensive care of the cancer patient: recent achievements and remaining challenges. Ann Intensive Care 2011;1:5.

9. Azoulay E, Lemiale V, Mokart D, Pene F, Kouatchet A, Perez $\mathrm{P}$, et al. Acute respiratory distress syndrome in patients with malignancies. Intensive Care Med 2014;40:1 106-14.

10. Azoulay E, Afessa B. The intensive care support of patients with malignancy: do everything that can be done. Intensive Care Med 2006;32:3-5.

11. Wohlfarth P, Staudinger T, Sperr WR, Bojic A, Robak O, Hermann A, et al. Prognostic factors, long-term survival, and outcome of cancer patients receiving chemotherapy in the intensive care unit. Ann Hematol 2014;93:1629-36.

12. Larche J, Azoulay E, Fieux F, Mesnard L, Moreau D, Thiery G, et al. Improved survival of critically ill cancer patients with septic shock. Intensive Care Med 2003;29:1688-95.

13. Azoulay E, Alberti C, Bornstain C, Leleu G, Moreau D, Recher $\mathrm{C}$, et al. Improved survival in cancer patients requiring mechanical ventilatory support: impact of noninvasive mechanical ventilatory support. Crit Care Med 2001;29:519-25.

14. Koutsoukou A. Admission of critically ill patients with cancer to the ICU: many uncertainties remain. ESMO Open 2017;2:e00105.

15. Niskanen M, Kari A, Halonen P. Five-year survival after intensive care: comparison of 12,180 patients with the general population. Finnish ICU Study Group. Crit Care Med 1996;24:1962-7.

16. Hermans G, Van Aerde N, Meersseman P, Van Mechelen H, Debaveye Y, Wilmer A, et al. Five-year mortality and morbidity impact of prolonged versus brief ICU stay: a propensity score matched cohort study. Thorax 2019;74:1037-45.

17. Thiery G, Azoulay E, Darmon M, Ciroldi M, De Miranda S, Levy V, et al. Outcome of cancer patients considered for intensive care unit admission: a hospital-wide prospective study. J Clin Oncol 2005;23:4406-13. 